\title{
Neoplasia Intra-epitelial Vulvar: Análise Clinicopatológica
}

Clinicopathologic Analysis of Vulvar Intraepithelial Neoplasia: Review of 46 Cases

Luiz Antonio Verdiani, Cássia Raquel Teatin Juliato, Sophie F. Mauricette Derchain, Júlio Eduardo Ferro

\section{RESUMO}

Objetivo: investigar alguns aspectos epidemiológicos, clinicos e patológicos dos vários graus de neoplasia intra-epitelial vilvar (NIV) e sua relação com o papilomavirus humano (HPV). Métodos: foram analisados os prontuários de 46 mulheres atendidas no Centro de Atenção Integral à Saúde da Mulher da Universidade Estadual de Campinas de janeiro de 1986 a

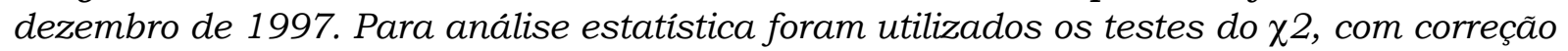
de Yates quando necessário, e exato de Fisher. Em relação à gravidade da lesão vulvar, seis mulheres apresentavam NIV 1, seis NIV 2 e 34 NIV 3.

Resultados: A idade, estado menstrual e idade da atividade sexual não estiveram relacionados com a gravidade da NIV, porém, as mulheres com mais de um parceiro sexual mostraram uma tendência maior a apresentar NIV $3(p=0,090)$. O tabagismo esteve significativamente associado à gravidade da lesão vulvar ( $p=0,031)$. O HPV foi mais freqüente nas mulheres com idade inferior a 35 anos $(p=0,005)$ e naquelas com múltiplas lesões ( $p=0,089)$. Embora o número não tenha mostrado relação com a gravidade da NIV ( $p=0,703)$, lesões maiores que $2 \mathrm{~cm}$ estiveram significativamente associadas com NIV 3 ( $p=0,009)$. O tratamento mais utilizado para NIV 3 foi cirúrgico, com exérese ou vulvectomia simples. Entre as oito mulheres que apresentaram recidiva, apenas uma era portadora de NIV 2.

Conclusões: Entre as mulheres com NIV, as fumantes e com mais de um parceiro sexual apresentaram lesões mais graves. A presença de HPV foi maior nas pacientes jovens com múltiplas lesões. Mulheres com NIV 3 apresentaram lesões maiores que $2 \mathrm{~cm}$ e uma alta taxa de recidiva, independentemente do tratamento utilizado.

PALAVRAS-CHAVE: Neoplasia intra-epitelial vulvar. Tabagismo.

Departamento de Tocoginecologia da Faculdade de Ciências Médicas da Universidade Estadual de Campinas - Área de Oncologia Ginecológica e Mamária.

Correspondência:
Luiz Antonio Verdiani

Rua Guido Pelliciari, 40

Torres de São José - Jundiaí - SP - CEP 13214-520

Fone: (011) 7392-5277 E-mail: laverdi@zaz.com.br 
Introdução

A neoplasia intra-epitelial vulvar (NIV) é um termo usado para designar alterações no epitélio escamoso da vulva com disturbio da maturação, formas celulares atípicas, mitoses e anormalidades nucleares como perda da polarização, desorganização da cromatina e irregularidade da membrana nuclear. Essas alterações podem ser classificadas em três graus, sendo denominadas NIV 1 quando atingem até um terço da extensão epitelial, NIV 2 quando afetam entre um e dois terços e NIV 3, também chamado de carcinoma in situ, quando envolvem mais de dois terços, chegando a acometer todo o epitélio $^{1,13}$.

Apesar do potencial evolutivo para lesões invasoras ser controverso, são consideradas precursoras do carcinoma vulvar ${ }^{1,8,11,12,13,19}$. São lesões raras, cuja incidência vem aumentando nos últimos vinte anos, principalmente em mulheres jovens. Nessas mulheres observa-se uma associação entre a NIV, o tabagismo e a infecção por papilomavírus humano (HPV), o que torna importante a avaliação de fatores epidemiológicos como idade ao início da atividade sexual e número de parceiros sexuais $^{1,2,8,11,12,13,19,22}$.

Dada as controvérsias em relação à evolução e ao acometimento de mulheres mais jovens, o tratamento das NIV passou a ser melhor avaliado, com o objetivo de preservar a anatomia e a atividade sexual. Atualmente o tratamento baseia-se em dois grandes grupos: a retirada cirúrgica da lesão ou sua destruição por métodos físicos ou químicos. Os que se fundamentam na exérese das lesões apresentam como grande vantagem a possibilidade de avaliação histológica dos tecidos, às custas de deformidades maiores como biópsia excisional, vulvectomia simples e total. A outra modalidade terapêutica consiste na destruição das lesões com métodos físicos como laser, eletro ou criocauterização, ou químicos como 5-fluorouracil, podofilina ou ácido tricloroacético. A desvantagem da destruição da lesão é a ausência de avaliação histológica, porém, com melhor preservação da anatomia vulvar. A escolha do método de tratamento depende não somente da idade da paciente, mas de outros fatores como a extensão da doença, a gravidade das atipias e a possibilidade de invasão focal $^{1,6,12,13,17,18,22}$.

Assim, o objetivo deste estudo foi investigar aspectos epidemiológicos, clínicos e histológicos dos vários graus de NIV e sua relação com alterações clínicas ou histológicas compativeis com a presença de HPV nas lesões.

\section{Pacientes e Métodos}

Este é um estudo clínico-descritivoretrospectivo, realizado por meio da avaliação dos prontuários de 46 mulheres com NIV atendidas no Centro de Atenção Integral à Saúde da Mulher (CAISM) da Universidade Estadual de Campinas (UNICAMP), de janeiro de 1986 a dezembro de 1997. Foram incluídas neste estudo as mulheres com prontuário completo, sem tratamento anterior da NIV e cujo exame anatomopatológico tenha sido realizado ou revisto pelo Departamento de Anatomia Patológica da Faculdade de Ciências Médicas (FCM)/UNICAMP. Foram excluídas as mulheres com carcinoma microinvasivo ou invasor da vulva. Foram avaliadas quanto à idade, cor, tabagismo, estado menstrual, idade quando do início da atividade sexual, número de parceiros, extensão da lesão e presença de alterações sugestivas de infecção por HPV no exame anatomopatológico em relação à gravidade da lesão vulvar. Para efeito de análise estatística, as pacientes com NIV $1(n=6)$ e $2(n=6)$ foram agrupadas e comparadas em conjunto $(n=12) \mathrm{com}$ aquelas com NIV $3(n=34)$. Também foram avaliados o tipo de tratamento e a taxa de recidiva nessas mulheres. Os dados foram coletados numa ficha pré-codificada, especialmente desenhada para este estudo, e inseridos em banco de dados em programa de computador Epi-info 6.02. Para análise estatística foram utilizados os testes do $\chi^{2}$, com correção de Yates quando necessário, Exato de Fisher e t de Student, com limite de confiança de $95 \%$.

\section{Resultados}

Em relação à gravidade da lesão vulvar, seis mulheres apresentavam NIV 1, seis NIV 2 e 34 NIV 3. Apesar do número de mulheres não-brancas ser muito pequeno, apenas cinco casos, as mulheres brancas apresentaram proporcionalmente mais NIV $3(78 \%)$, embora essa diferença não fosse estatisticamente significativa. A idade, estado menstrual e idade quando do início da atividade sexual não estiveram relacionados com a gravidade da lesão intra-epitelial, embora as mulheres com início da atividade sexual em idade inferior a 17 
anos apresentassem mais NIV 3. As pacientes com mais de um parceiro sexual mostraram uma tendência maior a apresentar NIV 3 quando comparadas às mulheres com apenas um parceiro $(p=0,090)$. O tabagismo esteve significativamente associado à gravidade da lesão vulvar: 93\% das mulheres fumantes apresentaram NIV 3, ao passo que apenas $59 \%$ das não-fumantes tinham essa graduação $(p=0,031)$. Embora o número de lesões não tenha relação com a gravidade da neoplasia intra-epitelial, lesões maiores que $2 \mathrm{~cm}$ estiveram significativamente associadas à NIV $3(p=0,009)$. Finalmente, 25 pacientes apresentavam alterações clínicas ou histológicas sugestivas de infecção por HPV, sem associação com a gravidade da lesão vulvar (Tabela 1).

Tabela 1 - Distribuição das pacientes segundo algumas variáveis epidemiológicas e a gravidade da lesão vulvar.

\begin{tabular}{|c|c|c|c|}
\hline Variável & $\underset{(n=12)}{\text { NIV } 1 \text { ou } 2}$ & $\begin{array}{l}\text { NIV } 3 \\
(n=34)\end{array}$ & $\mathrm{p}$ \\
\hline \multicolumn{4}{|l|}{ Cor } \\
\hline Branca & 9 & 32 & 0,103 \\
\hline Não branca & 3 & 2 & \\
\hline \multicolumn{4}{|l|}{ Idade } \\
\hline$\leq 35$ anos & 4 & 15 & \\
\hline$>35$ anos & 8 & 19 & 0,735 \\
\hline \multicolumn{4}{|c|}{ Estado menstrual } \\
\hline Menacme & 4 & 18 & \\
\hline Menopausa & 8 & 16 & 0,242 \\
\hline \multicolumn{4}{|c|}{ Inicio da atividade sexual } \\
\hline $\begin{array}{l}\leq 17 \text { anos } \\
>17 \text { anos }\end{array}$ & 4 & 18 & 0240 \\
\hline \multirow{2}{*}{\multicolumn{4}{|c|}{ Número de parceiros }} \\
\hline & & & \\
\hline$\leq \mathrm{um}$ & 10 & 18 & \\
\hline$>$ um & 2 & 16 & 0,090 \\
\hline \multicolumn{4}{|l|}{ Tabagismo* } \\
\hline $\mathrm{Sim}$ & 1 & 14 & \\
\hline Não & 11 & 16 & 0,031 \\
\hline \multicolumn{4}{|l|}{ HPV } \\
\hline Ausente & 5 & 16 & \\
\hline Presente & 7 & 18 & 0,747 \\
\hline \multicolumn{4}{|l|}{ Tamanho } \\
\hline$<2 \mathrm{~cm}$ & 12 & 20 & \\
\hline${ }^{3} 2 \mathrm{~cm}$ & 0 & 14 & 0,009 \\
\hline \multicolumn{4}{|c|}{ Número de lesões } \\
\hline Única & 4 & 8 & \\
\hline Múltipla & 8 & 26 & 0,703 \\
\hline
\end{tabular}

Essas alterações foram mais freqüentes nas mulheres com idade inferior a 35 anos $(p=0,005)$ e naquelas com lesões múltiplas $(p=0,089)$ (Tabela 2).
Tabela 2 - Distribuição das mulheres com NIV segundo a presença de HPV em relação à idade e número de lesões.

\begin{tabular}{lccr} 
Variáveis & Presente & HPV & Ausente \\
\hline $\begin{array}{l}\text { Idade } \\
\leq 35 \text { anos }\end{array}$ & 15 & & \\
$\geq 35$ anos & 10 & 17 & 0,005 \\
Média +DP & $38,2 \pm 17,5$ & $54,3 \pm 16,8$ & 0,0029 \\
& & & \\
Lesões & 4 & 8 & \\
$\quad$ Única & 21 & 13 & 0,089 \\
Múltiplas & 25 & 21 & \\
Total & & &
\end{tabular}

O tratamento mais utilizado para NIV 3 foi o cirúrgico, sendo realizada exérese da lesão com margens em 16 casos e vulvectomia simples em 13. Entre as 12 pacientes com NIV 1 ou 2 , apenas uma paciente com NIV 2, de 67 anos, foi submetida à vulvectomia. Entre os tratamentos químicos, três pacientes com NIV 1 ou 2 foram submetidas a aplicações tópicas de 5-flourouracil e em três pacientes com NIV 3 foram utilizadas aplicações tópicas de ácido tricloroacético, com uma recidiva. Entre as oito mulheres que apresentaram recidiva, apenas uma era portadora de NIV 2 (Tabela 3).

Tabela 3 - Distribuição das pacientes segundo a gravidade da lesão vulvar, o tipo de tratamento e a recidiva.

\begin{tabular}{lcc} 
Tratamento & $\begin{array}{c}\text { NIV 1 ou 2* } \\
\text { Recidiva/total }\end{array}$ & $\begin{array}{c}\text { NIV 3** } \\
\text { Recidiva/total }\end{array}$ \\
\hline Vulvectomia simples & $0 / 1$ & $2 / 13$ \\
Exérese & $1 / 6$ & $4 / 16$ \\
Tratamento químico & $0 / 3$ & $1 / 3$ \\
Total & $1 / 10$ & $7 / 32$ \\
\hline * Duas pacientes não foram tratadas. & \\
$* *$ Duas pacientes não voltaram para tratamento após a biópsia. \\
\hline
\end{tabular}

\section{Discussão}

A incidência de NIV tem apresentado um aumento constante, mais marcante nas duas últimas décadas. Esse aumento não se reflete na mesma proporção nos diagnósticos de carcinoma da vulva que, apesar de ter aumentado sua incidência de 3 para $8 \%$ das neoplasias femininas, continua raro. Entretanto, o câncer da vulva está sendo diagnosticado em mulheres mais jovens, o que pode ser reflexo do aumento da incidência de 
NIV observado nos últimos 20 anos entre mulheres jovens ${ }^{9,10}$.

Essa idéia é reforçada por estudos que demonstram uma correlação genética entre a VIN e o carcinoma da vulva, independentemente da presença do HPV, já que esse pode ser detectado em grande parte das NIV, mas apenas em uma pequena parcela dos carcinomas da vulva ${ }^{5,14}$. Porém, muitos consideram o HPV como importante fator etiológico na evolução das NIV para doença invasora, especialmente o tipo $16^{7,15,16,20}$.

Assim, como ocorre na maior parte dos relatos publicados na literatura, entre as mulheres com NIV estudadas, quase três quartos apresentavam NIV $3^{1,2,8,12,13}$. A maioria das mulheres com NIV 3 eram brancas. A NIV acomete preferencialmente pacientes jovens. Entretanto, apesar de ocorrer numa faixa etária mais precoce que o carcinoma invasor ${ }^{8,12,13}$, a idade e o estado menstrual não estavam relacionados com a gravidade da lesão intra-epitelial vulvar. Das 34 mulheres com NIV 3, 15 tinham idade inferior a 35 anos.

Existe uma clara associação entre câncer e tabagismo. Recentemente, o hábito de fumar tem sido relacionado com carcinoma do colo uterino ${ }^{21}$. Em relação à vulva os dados são pobres e conflitantes, embora sugiram que o hábito de fumar possa ser um importante cofator envolvido na etiologia de tumores vulvares, pricipalmente aqueles relacionados ao HPV e em mulheres jovens $\mathrm{s}^{4,7,10,13,19}$. Entre as mulheres aqui estudadas, $35,7 \%$ fumavam e o tabagismo esteve significativamente associado com a gravidade da lesão. O número de casos é insuficiente para demonstrar uma ação causal, porém, parece confirmar um potencial oncogênico do fumo sobre os tecidos epiteliais do trato genital inferior.

A maioria das lesões era múltipla, mostrando uma origem multicêntrica da doença. É provável que uma parte dos casos de NIV esteja associado com o HPV, principalmente em mulheres jovens ${ }^{1,4,7,8,11,12,16,17,19,20}$. A prevalência do vírus varia conforme a técnica utilizada para detecção, a idade e a gravidade da lesão: de forma geral as lesões histológicas HPV induzidas são descritas em 50\% das mulheres com NIV. Nos trabalhos baseados em alterações histológicas, a taxa de detecção do HPV é aproximadamente de 50\%. Porém, quando estudado numa população apenas de mulheres jovens, o HPV foi encontrado em até $78 \%$ dos casos ${ }^{3}$ e em lesões multicêntricas em até $96 \%$ dos NIV $3^{20}$. Nas mulheres avaliadas neste estudo, as alterações histológicas sugestivas de HPV estiveram presentes em $54 \%$ das pacientes.
A gravidade da lesão vulvar estava associada com a multiplicidade de parceiros, porém, não estava estatisticamente associada ao início precoce da atividade sexual e à presença do HPV. Por outro lado, mulheres com HPV associado à NIV apresentavam idade significativamente menor. $\mathrm{O}$ aumento da incidência de NIV em mulheres jovens, que tem sido observado nas duas últimas décadas, pode estar associado tanto a uma maior detecção de NIV 1 e 2 quanto a um possivel aumento da prevalência de HPV em mulheres sexualmente $\operatorname{ativas}^{1,4,5,7,8,12,13,19}$.

Há muita controvérsia relacionada ao tratamento ideal para NIV. Alguns sugerem que o tratamento de escolha para NIV 3 deveria ser a vulvectomia superficial total ou parcial, em virtude do potencial multicêntrico da patologia ${ }^{3}$, especialmente se for diagnosticada na menopausa, quando apresenta até $30 \%$ de possibilidade de evolução para carcinoma ${ }^{15}$. Porém, outros autores demonstram uma preferência em utilizar métodos menos agressivos, preservando a anatomia e a função vulvar, principalmente em mulheres jovens. A exérese local com margens e a destruição da lesão com laser são mais comumente utilizados ${ }^{1,4,13,22}$. De qualquer modo, a recorrência parece estar relacionada com outros fatores, tais como: idade, gravidade da lesão vulvar, multicentricidade e tabagismo ${ }^{13}$.

Entre as mulheres avaliadas neste estudo, a vulvectomia simples foi utilizada em 14 casos. Dessas, 13 eram mulheres mais idosas, apresentando NIV 3. Em apenas 6 casos foram utilizados métodos químicos. A principal desvantagem dos métodos químicos é que não é possivel uma análise histológica da lesão e a detecção de invasão inicial. Além disso, o tratamento causa desconforto local importante, exige um seguimento rigoroso e consultas médicas freqüentes. Observa-se, entretanto, melhor conservação vulvar e menor interferência na atividade sexual subseqüente. Esse é um aspecto importante a ser considerado em mulheres jovens.

Quanto à recidiva, oito das 46 mulheres estudadas apresentaram uma nova lesão vulvar após o tratamento. Sete dessas oito recidivas ocorreram em pacientes com NIV 3.

Concluímos que, entre as mulheres com NIV, as fumantes e com mais de um parceiro sexual apresentaram lesões mais graves. A presença de HPV foi maior nas pacientes jovens com lesões múltiplas. Mulheres com NIV 3 apresentaram lesões maiores que $2 \mathrm{~cm}$ e uma alta taxa de recidiva, independente do tratamento utilizado. 
moiety in some vulvar intraepithelial lesions and vulvar squamous cell carcinoma. Dermatol Surg 1997; 23:1025-8.

The purpose of the present study was to evaluate some epidemiological, clinical and pathological characteristics of the different grades of vulvar intraepithelial neoplasia (VIN), and its relation with the presence of human papillomavirus $(H P V)$. The charts of 46 women with VIN, examined from 1986 through 1997, were reviewed. For statistical analysis the $\chi^{2}$ with yates correction when appropriate, and Fisher's exact tests were used. Regarding the grade of VIN, six women presented VIN 1, six others had VIN 2 and the remaining 34 presented VIN 3. All women presented similar characteristics such as age, menstrual status and age at first sexual intercourse. Women with more than one lifetime sexual partner had a tendency to show more VIN $3(p=0.090)$. Cigarette smoking was significantly associated with the severity of the vulvar lesion $(p=0.031)$. HPV was significantly more frequent in women younger than 35 years of age $(p=0.005)$ and in women with multiple lesions $(p=0.089)$. Although the number of lesions were not related to the severity of VIN ( $p=0.703)$, lesions with extensions greater than $2 \mathrm{~cm}$ were significantly associated with VIN $3(p=0.009)$. The treatment of choice for VIN 3 was surgery, including local resection and simple vulvectomy. Eight women relapsed, and only one had VIN 2. We concluded that among women with VIN, cigarette smoking and more than one lifetime sexual partner were associated with high-grade lesions. HPV was more frequent among patients younger than 35 years of age presenting multiple lesions. Women with VIN 3 presented lesions bigger than 2 $\mathrm{cm}$ and a high relapse rate, despite the type of treatment applied.

KEY WORDS: Vulvar intraepithelial neoplasia. VIN. HPV. Smoking.

\section{Referências}

1.Barbero M, Micheletti L, Preti M, Cavanna L, Boselli F, Garuti G et al. Vulvar intraepithelial neoplasia. A clinicopathologic study of 60 cases. J Reprod Med 1990; 35:1023-28.

2.Benedet JL, Wilson PS, Matisic J. Epidermal thickness and skin appendage involvement in vulvar intraepithelial neoplasia. J Reprod Med 1991; 36:608-12.

3.Caglar H, Delgado, G, Hreshchyshyn MM. Partial and total skinning vulvectomy in treatment of carcinoma in situ of the vulva. Obstet Gynecol 1986; 68:504-7.

4.Edwards CL, Tortolero-Luna G, Linares AC, Malpica A, Baker VV, Cook E, et al. Vulvar intraepithelial neoplasia and vulvar cancer. Obst Gynecol Clin North Am 1996; 23:295-324.

5.Gao X, Chen HD, Wu X. Presence of human papillomavirus DNA and expression of L-fucose
6.Herod JJO, Shafi MI, Rollason TP, Luesley DM. Vulvar intraepithelial neoplasia with superficially invasive carcinoma of the vulva. Br J Obstet Gynaecol 1996; 103:453-6.

7.Hildesheim A, Han CL, Brinton LA, Kurman RJ, Schiller JT. Human papillomavirus type 16 and risk of preinvasive and invasive vulvar cancer: results from a seroepidemiological case-control study. Obstet Gynecol 1997; 90:748-54.

8.Italian Study Group on Vulvar Disease. Clinicopathologic analysis of 370 cases of vulvar intraepithelial neoplasia. J Reprod Med 1996; 41:665-70.

9.Iversen $\mathrm{T}$, Tretli S. Intraepithelial and invasive squamous cell neoplasia of the vulva: trends in incidence, recurrence and survival rate in Norway. Obstet Gynecol 1998; 91:969-72.

10.Jones RW, Baranyai J, Stables S. Trends in squamous cell carcinoma of the vulva: the influence of vulvar intraepithelial neoplasia. Obstet Gynecol 1997; 90:448-52.

11.Junge J, Poulsen H, Horn T, Hording U, Lundvall F. Human papillomavirus (HPV) in vulvar dysplasia and carcinoma in situ. APMIS 1995;103:501-10.

12.Kaufman RH. Intraepithelial neoplasia of the vulva. Gynecol Oncol 1995; 56:8-21.

13.Kuppers V, Stiller M, Somville T, Bender HG. Risk factors for recurrent VIN. Role of multifocality and grade of disease. J Reprod Med 1997; 42: 140-4.

14.Lin MC, Mutter GL, Trivijisilp P, Boynton KA, Sun D, Crum CP. Patterns of allelic loss (LOH) in vulvar squamous carcinomas and adjacent noninvasive epithelia. Am J Pathol 1998; 152:1313-8.

15.Paniel BJ, Haddad B, Berville-Levy S. Vulvar dysplasia. Rev Prat 1997; 47:1679-83.

16.Sarmiento JM, Wolff BG, Burgart LJ, Frizelle FA, Ilstrup DM. Perianal Bowen's disease: associated tumors, human papillomavirus, surgery and others controversies. Dis Colon Rectum 1997; 40:912-8.

17.Shafi MI, Luesley DM, Byrne P, Samra JS, Redman CW, Jordan JÁ, et al. Vulvar intraepithelial neoplasia- management and outcome. Br J Obstet Gynaecol 1989; 96: 1339-44.

18. Sillman FH, Sedlis A, Boyce JG. A review of lower genital intraepithelial neoplasia and the use of topical 5-fluorouracil. Obstet Gynecol Survey 1985; 40:190-220.

19.Trimble CL, Hildeshein A, Brinton L, Shah KV, Kurman RJ. Heterogeneous etiology of squamous carcinoma of the vulva. Obstet Gynecol 1996; 87:59-64.

20.van Beurden M, ten Kate FW, Tjong-A-Hung SP, de 
Craen AJ, van der Vange $\mathrm{N}$, Lammes $\mathrm{FB}$, et al. Human papillomavirus DNA in multicentric vulvar intraepithelial neoplasia. Int J Gynecol Pathol 1998; 17:12-6.

20.Winkelstein W. Smoking and cervical cancer-current sta- tus; a review. Am J Epidemiol 1990; 131:945-57.

21.Wright VC, Davies E. Laser surgery for vulvar intraepithelial neoplasia: principles and results. Am J Obstet Gynecol 1987,156:374-8.

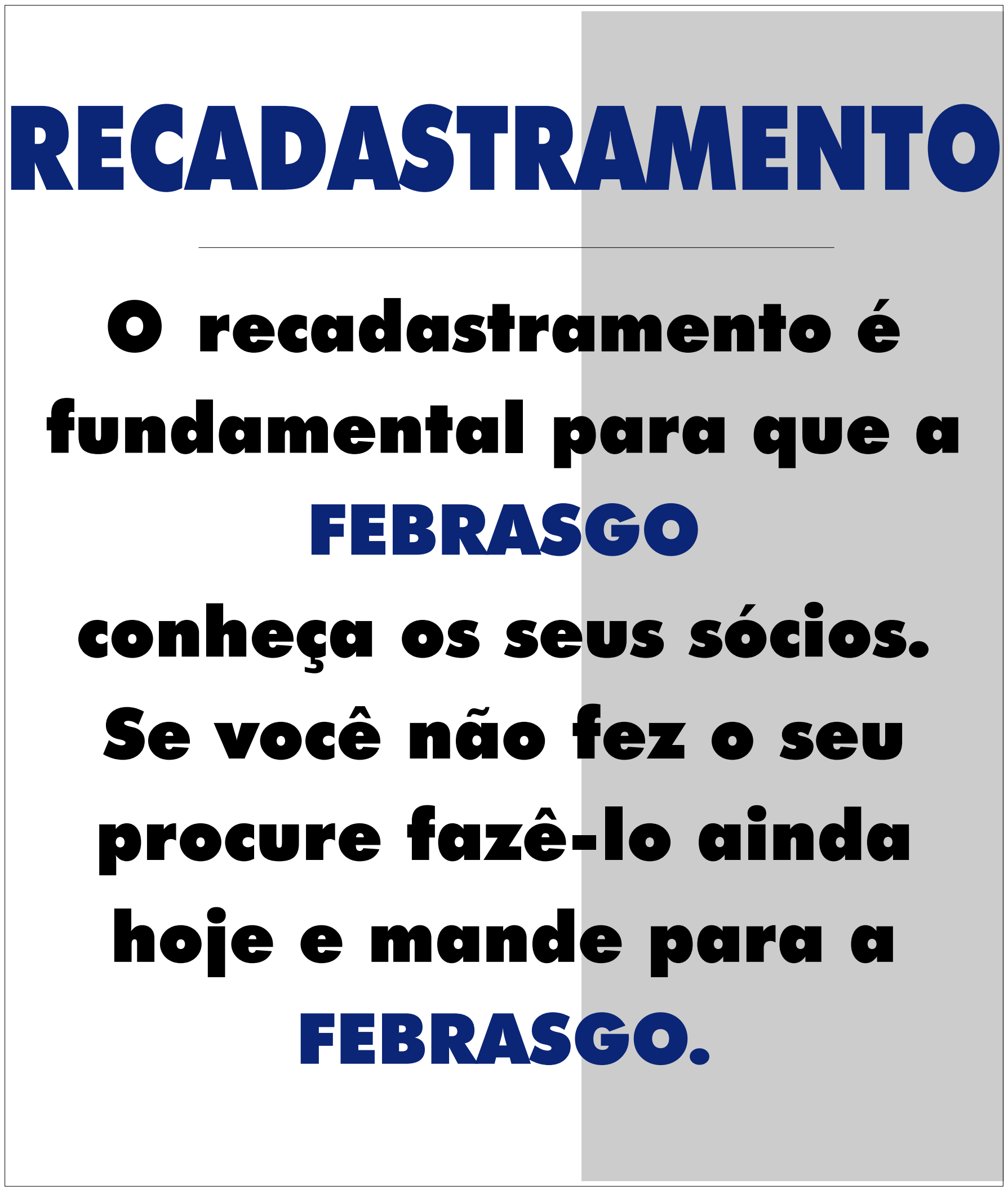

